\title{
Anatomical information for intercostobrachial nerve preservation in axillary lymph node dissection for breast cancer
}

\author{
J.J. Zhu ${ }^{1 *}$, X.F. Liu ${ }^{1}$, P.L. Zhang ${ }^{1}$, J.Z. Yang', J. Wang ${ }^{2}$, Y. Qin', \\ G.L. Zhang ${ }^{1}$, D.Q. Ren ${ }^{1}$, C.L. Cui ${ }^{3}$ and X.G. Guo ${ }^{1 *}$ \\ ${ }^{1}$ Department of Surgical Oncology, Cancer Hospital of Baotou City, \\ Baotou City, Inner Mongolia, China \\ ${ }^{2}$ Department of Anti-Cancer Association, Cancer Hospital of Baotou City, \\ Baotou City, Inner Mongolia, China \\ ${ }^{3}$ Department of Anatomy (Teaching and Research), Baotou Medical College, \\ Science and Technology University of Baotou, Baotou City, Inner Mongolia, \\ China
}

*These authors contributed equally to this study.

Corresponding author: X.G. Guo

E-mail: xiaoguangguocn@yeah.net

Genet. Mol. Res. 13 (4): 9315-9323 (2014)

Received May 29, 2013

Accepted October 25, 2013

Published January 24, 2014

DOI http://dx.doi.org/10.4238/2014.January.24.13

\begin{abstract}
This study aimed to provide additional anatomical information for axillary lymph node dissection (ALND) through in vivo anatomy studies of intercostobrachial nerve (ICBN) preservation in order to provide theoretical and practical experience for clinicians. A total of 156 patients with breast cancer underwent ALND at the Department of Gynecology of Baotou Tumor Hospital between June 2009 and March 2010. The origin, destination, main source, length, branch type, and direction of ICBN in axilla were observed, as well as its relationship with adjacent major blood vessels and nerves within the axilla. There were 120 cases of single trunk, 23 cases of double trunks, 9 cases of multiple trunks, and 4 cases without trunks in 156 patients with ICBN
\end{abstract}


preservation. The transverse diameter at the origin of the ICBN was 1.89 $\pm 0.44 \mathrm{~mm}$ with a length of $94.45 \pm 12.08 \mathrm{~mm}$; the distances were 77.19 $\pm 21.04 \mathrm{~mm}, 29.34 \pm 6.73 \mathrm{~mm}, 90.04 \pm 13.13 \mathrm{~mm}$, and $28.63 \pm 13.01$ $\mathrm{mm}$ from origin to the inferior margin at the midpoint of the clavicle, inferior margin of the axillary vein, the bottom of axilla, and branch point, respectively. The identification, dissection, and preservation of ICBN was simple and easy in a modified radical mastectomy for breast cancer and breast-conserving surgery, which only took 10-20 min, but effectively reduced the incidence of post-mastectomy pain syndrome and significantly improved the quality of life for patients after surgery.

Key words: Breast cancer; Axillary lymph node dissection (ALND); Intercostobrachial nerve (ICBN); Anatomy

\section{INTRODUCTION}

Breast cancer is one of the top three most common malignant tumors in females. Based on data collected by the World Health Organization (WHO), 1.4 million women suffered from breast cancer in 2006, and 0.4 million patients died of the cancer (Smigal et al., 2006). In China, the mortality and morbidity of breast cancer has increased annually, and there is now 1 death every $12 \mathrm{~min}$ (Peng et al., 2013) and more than 40 in 100,000 women are diagnosed with breast cancer per year in some cities (Leidenius et al., 2003; Zhao et al., 2013). Even worse, the morbidity of breast cancer has been increasing rapidly, by about 3 to $4 \%$ every year (Yan et al., 2013). At the same time, the age of the breast cancer patients has become increasingly younger, and multiple researchers have found that the younger the patient is at the time of diagnoses, the poorer the prognosis for that patient (Benton et al., 2014). Although an early diagnosis results in earlier treatment, the mortality of women diagnosed before the age of 45 was higher than for women diagnosed when older (González Jiménez et al., 2012).

Early-stage breast cancer can be cured in more than $60 \%$ of patients (Lu and Labhasetwar, 2013), and surgical treatment is still one of the most important interventions. Recently, due to increasing attention on the quality of life and research advances on the biological characteristics of breast cancer, breast cancer surgery has focused more on the recovery of the patients. Accordingly, the focus is not only to remove the tumor and metastatic lymph node, but also to protect the function of the surrounding tissues.

Modern breast cancer surgery has evolved from the traditional radical operations, extensive radical mastectomy, modified radical mastectomy (Patey-Dyson operation: the pectoralis minor muscle is resected and the pectoralis major muscle is preserved; Auchincloss operation: both the pectoralis minor muscle and the pectoralis major muscle are preserved), and breast-conserving surgery for early breast cancer, to now include radical mastectomy for early-stage breast cancer and stage I breast reconstruction. Breast-conserving surgery is well accepted in foreign countries, while modified radical mastectomy is the main surgical procedure in China. However, axillary lymph node dissection (ALND) is necessary in surgery for breast cancer, irrespective of the type of operation that is elected.

Resection or damage to the intercostobrachial nerve (ICBN) during ALND would limit the activities of the upper limbs of patients after surgery and causes post-mastectomy 
pain syndrome, such as pain, numbness, and sensory disturbances in the lateral thoracic wall, axilla, and arms, which occur in 18-50\% of patients (McIntosh and Purushotham, 1998; Carpenter et al., 1999; Bergmann et al., 2012; Kodama et al., 2012; Taira et al., 2014) and seriously reduces the postoperative quality of life for patients with breast cancer. Therefore, an increasing number of surgeons have considered improvements to surgical procedures to protect the ICBN during ALND.

Our study cohort included 156 patients with breast cancer who had undergone ALND between June 2009 and March 2010, and the occurrence rate, composition, branch type, and main lines of ICBN were explored, as was the anatomical location of the adjacent blood vessels, nerves, and muscle tissue, in order to provide additional clinical applied anatomy data for ICBN preservation in ALND.

\section{MATERIAL AND METHODS}

\section{General clinical data}

Our study included 156 patients with breast cancer, ranging in age from 31 to 90 years, with a median age of 49.8 years. There were 89 cases of cancer in the left breast and 67 cases of cancer in the right breast, including 89 cases in stage I, 51 cases in stage II, and 16 cases in stage III. The pathological type of tumors included: 123 cases of invasive ductal carcinoma, 22 cases of invasive lobular carcinoma, 3 cases of cephaloma, 1 case of tubular carcinoma, 2 cases of glycogen-rich clear cell breast carcinoma, 3 cases of mucinous carcinoma, and 2 cases of Peget's disease complicated with invasive ductal carcinoma. This study was conducted in accordance with the Declaration of Helsinki and with approval from the Ethics Committee of Baotou Tumor Hospital. Written informed consent was obtained from all participants.

\section{Surgical methods}

Breast-conserving surgery (extensive tumor resection with ALND) was performed in 65 patients and modified radical mastectomy (Auchincloss operation: both the pectoralis minor muscle and pectoralis major muscle are preserved) was conducted in 91 patients.

\section{Observational methods}

A total of 156 patients with breast cancer underwent ALND. The main branch type, source composition, length, and direction of ICBN in axilla were observed and recorded, as was its relationship with important adjacent structures within the axilla.

The main lines of ICBN and the distances from the origin to important structures, such as the inferior margin at midpoint of the clavicle and the inferior margin of the axillary vein, were observed and recorded in 118 patients. We defined anatomical distances as the following: nerve length, distance from the origin to the destination; distance to the inferior margin at midpoint of the clavicle, distance from the origin to the inferior margin at the midpoint of the clavicle; distance to the inferior margin of the axillary vein, distance from the origin to the inferior margin of the axillary vein; distance to the bottom of axilla, distance from the origin to the bottom of axilla; and distance to the branch point, distance from the origin to the branch point. 


\section{Statistical analyses}

All data were analyzed using the SPSS 13.0 software.

\section{RESULTS}

\section{Occurrence rate of ICBN}

Among 156 surgeries, there were 152 cases with ICBN and 4 cases without ICBN, representing 97.44 and $2.56 \%$ of our total cases, respectively.

\section{Main ICBN and its branch types}

Among 152 cases with ICBN, there were 120 cases of single trunk, 23 cases of double trunks, and 9 cases of multiple trunks (Table 1).

\begin{tabular}{|c|c|c|c|}
\hline Branch type & Source & Cases & Percentage $(\%)$ \\
\hline \multicolumn{4}{|l|}{ Single trunk } \\
\hline \multirow[t]{2}{*}{ Single trunk without branches } & Originate from the second intercostal nerve & 25 & 16.04 \\
\hline & Originate from the first intercostal nerve & 2 & 1.28 \\
\hline Single trunk with two branches & Originate from the second intercostal nerve & 73 & 46.79 \\
\hline Single trunk with multi-branches & Originate from the second intercostal nerve & 20 & 12.82 \\
\hline \multicolumn{4}{|l|}{ Double trunks } \\
\hline \multirow[t]{2}{*}{ Double trunks with two branches } & Originate from the first and second intercostal nerves & 8 & 5.13 \\
\hline & Originate from the second and third intercostal nerves & 2 & 1.28 \\
\hline \multirow[t]{2}{*}{ Double trunks with multi-branches } & Originate from the first and second intercostal nerves & 9 & 5.77 \\
\hline & Originate from the second and third intercostal nerves & 4 & 2.56 \\
\hline Multiple trunks & Originate from the first, second and third intercostal nerves & 9 & 5.77 \\
\hline No trunks & & 4 & 2.56 \\
\hline
\end{tabular}

\section{Sources of ICBN}

There were 2 cases of single trunk that originated from the first intercostal nerve, 118 cases that originated from the second intercostal nerve, 17 cases of double trunks that originated from the first and second intercostal nerves, 6 cases from the second and third intercostal nerves, and 9 cases of multiple trunks that originated from the first, second, and third intercostal nerves.

\section{Direction and adjacent relationships of ICBN}

All single trunks of ICBN that originated from the second intercostal nerve extended to the second intercostal muscles from the posteromedial side of the inferior margin of the pectoralis minor muscle. It almost vertically passed the long thoracic nerve, lateral thoracic artery, and the front of thoracodorsal nerve and vessel, and it approximately paralleled with the axillary vein, crossed the axillary loose connective tissue, passed through the front of latissimus dorsi, and was distributed in the skin at its adnexa of the inferior margin. All branches of ICBN originated from the lateral thoracodorsal nerve and vessel. A single trunk of the ICBN 
that originated from the first intercostal nerve in 2 cases was adjacent to the axillary sheaths and went along the back of the thoracodorsal vessel, thoracodorsal nerve, and long thoracic nerve. One branch of double trunks that originated from the first intercostal nerve in 3 cases passed along the front of thoracodorsal vessel, thoracodorsal nerve, long thoracic nerve, and lateral thoracic vessel; all of the others extended along the back of the thoracodorsal vessel, thoracodorsal nerve, long thoracic nerve, and lateral thoracic vessel. The orientation of multitrunk ICBN was complex, which crossed with the thoracodorsal vessel, thoracodorsal nerve, long thoracic nerve, and lateral thoracic vessel.

\section{Distribution of ICBN}

ICBN was found to be mainly distributed in the skin at the posteromedial side of arm and bottom of the axillary and lateral thoracic walls, but different types had distinct control scopes. Two cases of single trunk originated from the first intercostal nerve and 25 cases of single trunk without branches that originated from the second intercostal nerve and directly reached the medial arm; the thicker upper branch in the single trunk with branches was distributed at the medial arm and the bottom of the axillary, while the thinner lower branch spread at the lateral thoracic wall; the upper branch in double and multiple trunks was distributed at the bottom of the axillary and the medial arm, while the lower branch spread at the lateral thoracic wall and part was distributed at the medial arm and the bottom of the axillary. During surgery, ICBN was found to be linked with the medial brachial cutaneous nerve in 123 cases $(80.92 \%$ of ICBN).

\section{Measurement of ICBN}

The ICBN was only measured in 118 patients due to the limitation of study objective conditions. In addition, only the part between the intercostal muscle and terminal branch that entered into the medial arm or subcutaneous axillary were measured to avoid unnecessary damage in patients, as shown in Table 2.

\begin{tabular}{|c|c|c|c|}
\hline Parameters (in mm) & Maximum & Minimum & Mean $\pm \mathrm{SD}$ \\
\hline Transverse diameter at origin & 1.1 & 2.9 & $1.89 \pm 0.44$ \\
\hline Nerve length & 74.9 & 142.3 & $94.45 \pm 12.08$ \\
\hline Distance from origin to the inferior margin at midpoint of the clavicle & 45.8 & 112.0 & $77.19 \pm 21.04$ \\
\hline Distance from origin to inferior margin of axillary vein & 9.8 & 45.7 & $29.34 \pm 6.73$ \\
\hline Distance from origin to the bottom of axilla & 69.6 & 142.3 & $90.04 \pm 13.13$ \\
\hline Distance from origin to branch point & 9.2 & 66.1 & $28.63 \pm 13.01$ \\
\hline
\end{tabular}

\section{DISCUSSION}

The lateral cutaneous branch of the second intercostal nerve divides into a thinner or absent anterior branch and a thicker posterior branch that is generally considered as the ICBN (Cunnick et al., 2001). In some conditions, the ICBN combines with the lateral cutaneous branch of the first and/or intercostal nerve (Loukas et al., 2006). Our study found that a single trunk of the ICBN that originated from the second intercostal nerve accounted for $75.6 \%$ $(118 / 156)$ of cases, but in $21.8 \%$ of cases, the ICBN originated from the first intercostal nerve, 
the first and second intercostal nerves, the second and third intercostal nerves, or the first, second, and third intercostal nerves. Secondly, in $2.6 \%$ of cases, the ICBN was absent (4/156). We found that the percentage of the first intercostal nerve was $17.9 \%(28 / 156)$, which was similar to the report from Benton et al. (2014) that approximately $80 \%$ of the first intercostal nerve had no lateral cutaneous branch, but they also showed that the lateral cutaneous branch of $30 \%$ of the third intercostal nerve was involved in ICBN, which was different from the 9.6\% (15/156) revealed in our research. This discrepancy might be associated with different subjects, anatomy, and observation methods and resolving this will require further investigation.

All single trunks of ICBN that originated from the second intercostal nerve extended to the second intercostal muscles from the posteromedial side of the inferior margin of the pectoralis minor muscle, almost vertically passed the long thoracic nerve, lateral thoracic artery, and the front of thoracodorsal nerve and vessel, approximately paralleled with axillary vein, crossed axillary loose connective tissue, passed through the front of latissimus dorsi, and was distributed in the skin at its adnexa of the inferior margin. All branches of ICBN originated from the lateral thoracodorsal nerve and vessel. A single trunk of ICBN that originated from the first intercostal nerve in 2 cases was adjacent to the axillary sheaths and went along the back of thoracodorsal vessel, thoracodorsal nerve, and long thoracic nerve. One branch of double trunks that originated from the first intercostal nerve in 3 cases passed along the front of the thoracodorsal vessel, thoracodorsal nerve, long thoracic nerve, and lateral thoracic vessel; all the others extended along the back of thoracodorsal vessel, thoracodorsal nerve, long thoracic nerve, and lateral thoracic vessel. The orientation of multi-trunk ICBN was complex, which crossed with the thoracodorsal vessel, thoracodorsal nerve, long thoracic nerve, and lateral thoracic vessel. This was basically consistent with the autopsy results reported previously (Torresan et al., 2003).

In our research, 2 cases of single trunk that originated from the first intercostal nerve and 25 cases of single trunk without branches that originated from the second intercostal nerve directly reached the medial arm; the thicker upper branch in the single trunk with branches was distributed throughout the medial arm and the bottom of the axillary, while the thinner lower branch was spread at the lateral thoracic wall; the upper branch in double and multiple trunks was distributed at the bottom of the axillary and medial arm, while the lower branch was spread at the lateral thoracic wall and partially distributed at the medial arm and bottom of the axillary. These results were different from what Taira et al. (2014) reported, for whom almost all of the upper branch or trunk of the lateral cutaneous branch of the second intercostal nerve dominated the bottom of the axillary and medial arm. Resolving these differences will require further research. In addition, we found that $80.9 \%$ of medial brachial cutaneous nerves were linked with ICBN, which was similar to the $83.8 \%$ reported by Kasumi (2005). Moreover, approximately all of the medial brachial cutaneous nerves were linked with the lateral cutaneous branch of the second intercostal nerve. Additionally, the diameters of the two nerves were negatively correlated, which demonstrated that they were complementary in morphology and function, and thereby formed a complete neural network to control skin and hypodermis of upper limb, lateral thoracic wall, and axilla.

Resection or damage to the ICBN in ALND limits the activities of the upper limbs of patients after surgery and causes post-mastectomy pain syndrome, such as pain, numbness, and sensory disturbance in the lateral thoracic wall, axilla, and arms; this occurs in $18-50 \%$ of patients and has been documented by many studies (Paredes et al., 1990; Wong, 2001; Freeman et al., 2003) to seriously influence the postoperative quality of life for patients with 
breast cancer. The pain is persistent in most cases and rarely resolves spontaneously or by conventional analgesia (McIntosh and Purushotham, 1998; Carpenter et al., 1999; Kodama et al., 2012). Why does ICBN damage cause ICBN syndrome? Currently, it is known that the ICBN is cut-off and its stump develops neuroma. In 2001, Wong opened and explored the original surgical area in 5 breast cancer patients with postoperative pain and discovered intercostal neuromas in all of these patients who were relieved from pain after the intercostal neuromas were resected. Furthermore, ICBN syndrome is mainly responsible for the chronic discomfort in patients with breast cancer after surgery due to the poor efficacy of drugs for neuropathic pain (Carpenter et al., 1999). Paredes et al. (1990) followed up patients with breast cancer for 3 years after surgery and found that ICBN preservation did not affect the long-term survival of patients. The patients in our study were followed up for 1 to 9 months and no local recurrences or metastases were observed, which was consistent with the literature. There were no distinct differences in surgical time, blood loss, or the number of removed lymph nodes between the ICBN preservation group and ICBN resection group, which is similar to what van Dam et al. (1993) reported.

In modified radical mastectomy, the inferior margin of the pectoralis minor muscle should be confirmed before the axillary lymph nodes are cleared up, followed by processing of the lateral thoracic wall. According to our research, ICBN pierced the thoracic wall at the deep side of the inferior margin of the pectoralis minor muscle that was 4-12 cm away from the inferior margin at the midpoint of the clavicle. Therefore, it was critical to search for and dissect the ICBN in this region, and the lateral thoracic wall could be handled audaciously out of this region. Kasumi (2005) found that $89.1 \%$ of sentinel lymph nodes were located 2 $\mathrm{cm}$ outside of the head end of ICBN and the exterior margin of the pectoralis minor muscle, and the ICBN could be exposed by dissecting downwards from the axillary artery and vein in a layer-by-layer manner. We thought that it was better to identify the ICBN from the lateral thoracic wall because the initiation site of the ICBN was relatively fixed and the nerve was thicker; however, the ICBN had various sources, trunks, and branch types and, thus, exposure of the ICBN from the origin could reduce damage to the ICBN trunk and branches to the maximum extent. Therefore, it was possible to injure the ICBN during dissection of the lateral pectoral nerve and vessel and resection of the axillary lymph nodes, which should be noted by clinicians.

In breast-conserving surgery, the skin incision for axillary lymph node resection was different from that of breast resection and enlargement of the surgical field, which facilitated the processing of the lateral thoracic wall. Therefore, the long skin incision at axilla was made from the exterior margin of the pectoralis major muscle to the anterior margin of the latissimus dorsi, where the subcutaneous tissue was completely separated and the axillary fascia was totally exposed. The exterior margin of the pectoralis major muscle was exposed downwards as far as possible before further procession of the lateral thoracic wall. Then, the ICBN was carefully identified and dissected according to a previously described method.

After the ICBN was dissected from the lateral thoracic wall, towel and rat forceps were utilized to lift and discreetly isolate the ICBN along the nerve until the entire trunk was exposed. When the ICBN was close to the inferior margin of latissimus dorsi, a retractor was used to pull it to the head side and clear downwards from the edge of the axillary vein. During this procedure, a traction belt could not be pulled strongly and the electrotome could be close to the nerve. In addition, ICBN entered the arm from different positions, thus it was better to 
confirm the direction of the ICBN when clearing the lateral axillary lymph nodes. Moreover, ICBN entered the arm in a fan shape in some cases, which required careful isolation so as not to waste the previous efforts.

The lateral cutaneous branch of the second intercostal nerve was the main source of the ICBN, accounting for $75.6 \%(118 / 156)$ of cases. There were $2.6 \%$ of patients without an ICBN (4/156).

According to the main trunk and branches, ICBNs could be divided into single trunk without branches, single trunk with two branches, single trunk with multi-branches, or double trunks with two branches and multiple trunks.

The identification, dissection, and preservation of ICBN was simple and easy in modified radical mastectomy for breast cancer and breast-conserving surgery, which only took 10-20 min, but effectively reduced the incidence of post-mastectomy pain syndrome and significantly improved the quality of life for patients after surgery.

\section{REFERENCES}

Benton MJ, Schlairet MC and Gibson DR (2014). Change in quality of life among breast cancer survivors after resistance training: is there an effect of age? J Aging Phys. Act. 22: 178-185.

Bergmann A, Mendes VV, de Almeida DR, do Amaral E Silva, et al. (2012). Incidence and risk factors for axillary web syndrome after breast cancer surgery. Breast Cancer Res Treat. 131: 987-992.

Carpenter JS, Sloan P, Andrykowski MA, McGrath P, et al. (1999). Risk factors for pain after mastectomy/lumpectomy. Cancer Pract. 7: 66-70.

Cunnick GH, Upponi S and Wishart GC (2001). Anatomical variants of the intercostobrachial nerve encountered during axillary dissection. Breast 10: 160-162.

Freeman SR, Washington SJ, Pritchard T, Barr L, et al. (2003). Long term results of a randomised prospective study of preservation of the intercostobrachial nerve. Eur. J. Surg. Oncol. 29: 213-215.

González Jiménez E, García López PA, Schmidt-Río-Valle J and Valenza C (2012). Influence of nutritional status, hormones serum levels, and family history on breast cancer development. Rev. Med. Chil. 140: 1263-1267.

Kasumi F (2005). Breast Surgery Points and Blind Spots. In: Translator-in-chief (Duan ZY, ed.). Liaoning Science and Technology Press, Shenyang, 186-188.

Kodama H, Mise K and Kan N (2012). Partial lower axillary dissection for patients with clinically node-negative breast cancer. J. Int. Med. Res. 40: 2336-2345.

Leidenius M, Leppanen E, Krogerus L and von Smitten K (2003). Motion restriction and axillary web syndrome after sentinel node biopsy and axillary clearance in breast cancer. Am. J. Surg. 185: 127-130.

Loukas M, Hullett J, Louis RG Jr, Holdman S, et al. (2006). The gross anatomy of the extrathoracic course of the intercostobrachial nerve. Clin. Anat. 19: 106-111.

Lu S and Labhasetwar V (2013). Drug Resistant Breast Cancer Cell Line Displays Cancer Stem Cell Phenotype and Responds Sensitively to Epigenetic Drug SAHA. Drug Deliv. Transl. Res. 3: 183-194.

McIntosh SA and Purushotham AD (1998). Lymphatic mapping and sentinel node biopsy in breast cancer. Br. J. Surg. 85: 1347-1356.

Paredes JP, Puente JL and Potel J (1990). Variations in sensitivity after sectioning the intercostobrachial nerve. Am. J. Surg. 160: 525-528.

Peng Q, Zhao L, Hou Y, Sun Y, et al. (2013). Biological characteristics and genetic heterogeneity between carcinomaassociated fibroblasts and their paired normal fibroblasts in human breast cancer. PLoS One 8: e60321.

Smigal C, Jemal A, Ward E, Cokkinides V, et al. (2006). Trends in breast cancer by race and ethnicity: update 2006. $C A$ Cancer J. Clin. 56: 168-183.

Taira N, Shimozuma K, Ohsumi S, Kuroi K, et al. (2014). Impact of preservation of the intercostobrachial nerve during axillary dissection on sensory change and health-related quality of life 2 years after breast cancer surgery. Breast Cancer 21: 183-190.

Torresan RZ, Cabello C, Conde DM and Brenelli HB (2003). Impact of the preservation of the intercostobrachial nerve in axillary lymphadenectomy due to breast cancer. Breast J. 9: 389-392.

van Dam MS, Hennipman A, de Kruif JT, van der Tweel I, et al. (1993). Complications following axillary dissection for breast carcinoma. Ned. Tijdschr. Geneeskd. 137: 2395-2398.

Genetics and Molecular Research 13 (4): 9315-9323 (2014) 
Wong L (2001). Intercostal neuromas: a treatable cause of postoperative breast surgery pain. Ann. Plast. Surg. 46: 481484.

Yan M, Lü HM, Liu ZZ, Liu H, et al. (2013). High risk factors of brain metastases in 295 patients with advanced breast cancer. Chin. Med. J. 126: 1269-1275.

Zhao L, Li P, Li F, Yang Y, et al. (2013). The prognostic value of circulating tumor cells lacking cytokeratins in metastatic breast cancer patients. J. Cancer Res. Ther. 9: 29-37. 